\title{
Modeling Jupiter's Synchrotron Emission from Relativistic Electron Trapped in Jovian Magnetosphere
}

\author{
Shubha Singh ${ }^{1, *}$, A. K. Singh ${ }^{2}$ and R.P. Singh ${ }^{2}$
}

${ }^{1}$ Department of Physics, College of Eng. Science, University of Petroleum and Energy Studies, Dehradun-248007, Uttarakhand, India

${ }^{2}$ Atmospheric Research Lab, Department of Physics, Banaras Hindu University, Varanasi-221005, India

\begin{abstract}
The relativistic electron energy in few $\mathrm{MeV}$ range, trapped in the Jovian magnetosphere emit electromagnetic waves in wide radio frequency domain which escapes the generation region and propagates towards the Earth's atmosphere. The flux density of the emission is a function of the electron distribution (spatial and angular), the energy spectrum of the electron, the magnetic field strength and configuration. The measured spectral power density of high energetic electrons and its distribution with frequency can be used to find out the distribution of relativistic electrons in the inner magnetosphere having the range $L_{J}=3$ Jovian radius. In present communication the variation of radiated power per electron with energy through the synchrotron radiation process have been shown and discussed. The simulated flux density is compared with corresponding recent emitted radio spectrum data available from Cassinni flyby. The investigation enables us to explain the mechanism and characteristic properties of Jovian magnetosphere.
\end{abstract}

\section{Introduction}

In 1950's, radio emissions from the Jupiter were recorded in two frequency bands referred as decametric (DAM) and decimetric (DIM). The observed decametric emissions were sporadic in character, and confined to frequencies less than $40 \mathrm{MHz}$ [1] and are attributed to electron cyclotron maser emissions, emitted by $\mathrm{keV}$ electrons in the Jupiter's auroral region. The non-thermal decimetric radiation was first detected by ground-based radio telescopes in the late 1950's and later attributed to synchrotron radiation from relativistic electrons trapped in the tilted dipole field of the Jovian magnetosphere [2-4]. It has been proposed that the emissions originated from the Jupiter's inner radiation belt $\left(\mathrm{L}_{\mathrm{I}} \leq 4\right)$. The generation region is supposed to be located near the magnetic equator and the high magnetic latitudes [5]. Analyzing features of the observed decametric bursts, Warwick [6] suggested that the source of decametric emission is outside the planet and the magnetic field helps in focusing these radiations [5]. Singh et al.[7] studied the detailed features of synchrotron radiation using the Pioneer-10 data for electrons and magnetic field. Using tilted magnetic field geometry, they have schematically shown the reception of Jupiter's emissions on the surface of the Earth. De Pater [8] presented a schematic representation of wave-spectrum observed from the Jupiter displaying the intensity and frequency range from a few $\mathrm{MHz}$ up to a few tens $\mathrm{GHz}$. The same is redrawn in Figure 1. It is shown that at frequencies $\geq 5$ $\mathrm{GHz}$, thermal emissions dominate total emissions. However, still there is a significant contribution from the planet's synchrotron radiation. The contribution from the thermal emission and synchrotron emission can be separated by measuring polarization/imaging the planet at high angular resolution $[9,10]$. Synchrotron radiation between $74 \mathrm{MHz}$ and $15 \mathrm{GHz}$ has been imaged by using telescope array [11]. Using the Cassini spacecraft data, Bolton et al. [12] presented the observation of synchrotron radiation at $13.8 \mathrm{GHz}$, which confirms the presence of electrons with energies up to $50 \mathrm{MeV}$ or may be more. The observation by Very Large Array at still higher frequencies implies the presence of electrons with energies $\geq 100 \mathrm{MeV}$ de Pater and Kloosterman [10,13] presented the observations of the synchrotron radiation from Jupiter obtained in July 2004 with the Very Large Array at a frequency of 15 $\mathrm{GHz}$. The recent analysis and understanding of the Jovian synchrotron radio emission with a radiation-belt model is presented by SantosCosta and Bolton [13]. Recently Ezoe et al. [14] reported the discovery of diffused hard (1-5 keV) X-ray emissions around Jupiter in a deep
Suzuku X-ray imaging spectrometer data. Horne et al. [15] suggest that gyro-resonant electron acceleration is an important part of a multistep process to produce Jupiter's synchrotron radiation. They further suggested that the Gyroresonant acceleration is most effective between 6 and 12 Jovian radii and provides the missing step in the production of intense synchrotron radiation from Jupiter. Recently Tsuchiya et al. [16] studied the total flux density of Jupiter's synchrotron radiation at $325 \mathrm{MHz}$ with the Iitate Planetary Radio Telescope to investigate short-term variations in Jupiter's radiation belt with a time scale of a few days to a month. They reported that total flux density showed a series of short-term increases and subsequent decreases. De Pater [8] suggested that the Jupiter's synchrotron radiation is concentrated near the magnetic equator, with two weaker rings of emissions at higher latitudes. Measurements of flux density of Jovian radio spectrum in September 1998 are found to decrease at lower frequencies as compared to that of June 1994 [10]. This has been suggested to be due to pitch angle scattering, coulomb scattering, and energy degradation by dust particle etc. Since these processes are relatively more effective for the low-energy electrons. The charged particles present in the presence of the Jupiter's magnetic field are accelerated by the plasma waves present in the medium. The mapping of distribution of synchrotron emission may provide an understanding of highly energetic electron distribution in the region of radiation belt provided magnetic field structure is known. The synchrotron radiation received at the Earth's surface is a consequence of the combined effects of several factors such as the beaming effect, polarization and rocking effect due to the Jovian \pm 100 tilted axis. It has been an important tool for understanding the magnetic field and relativistic electron population in the inner (1.2-3.5 Jovian

*Corresponding author: Shubha Singh, Asst. Professor Department of Physics, CoES UPES (University of Petroleum and Energy Studies) Dehradun-248007, India, Tel: 0135-2770137; 2776061; E-mail: singhshubhabhu@gmail.com

Received October 17, 2015; Accepted December 03, 2015; Published December 11, 2015

Citation: Singh S, Singh AK, Singh RP (2015) Modeling Jupiter's Synchrotron Emission from Relativistic Electron Trapped in Jovian Magnetosphere. Hydrol Current Res 7: 218. doi:10.4172/2157-7587.1000218

Copyright: ( 2015 Singh S, et al. This is an open-access article distributed under the terms of the Creative Commons Attribution License, which permits unrestricted use, distribution, and reproduction in any medium, provided the original author and source are credited. 


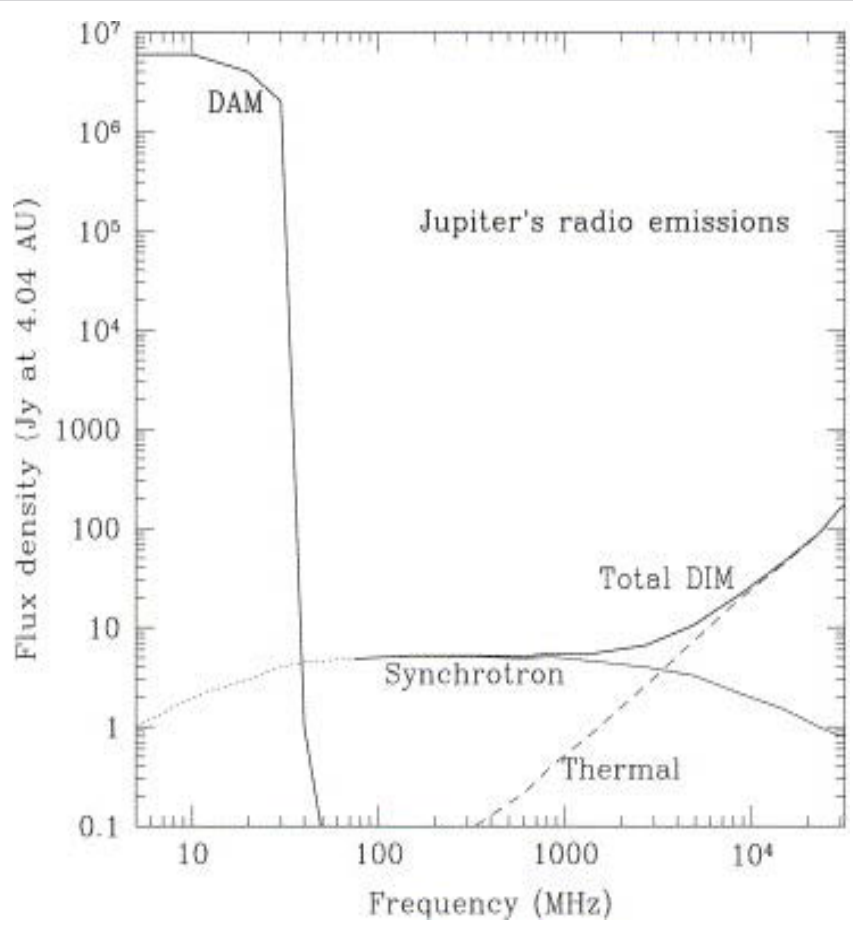

Figure 1: The observed radio spectrum from Jupiter exhibiting frequency from a few $\mathrm{MHz}$ up to a few tens $\mathrm{GHz}$ (de Pater, 2004).

radii) Jovian magnetosphere. The Jovian's synchrotron radiation is seen at the Earth above the galactic noise level due to its strong magnetic field (4.28 gauss at the equator) and the typical magnetic field strength at Jupiter's surface ranges from 2-14 gauss. The observed equatorial and high-latitude emission has been modeled with a combination of an anisotropic and isotropic electron population trapped in the Jupiter's inner radiation belt $[17,18]$. Such modeling studies may also provide new information about high order magnetic moments of the Jovian magnetic field. These waves have been used as a non-destructive probe of the inner magnetosphere because the received signal did not have significant deformation while propagating from the Jupiter's surface to the Earth's surface.

Thorne [19] developed a theoretical model of the synchrotron radiation with a dipole magnetic field and calculated the total power in the synchrotron radiation over the entire dipole field. Westfold [20] investigated the polarization and spectral distribution of the synchrotron radiation and showed that degree of polarization increases with frequency which is in conformity with observation. De Pater [21,22] presented a model calculations of Jupiter's synchrotron radiation based on the radio data and systematically analyzed the high energy electron's distributed in the Jupiter's inner magnetosphere and also the east-west asymmetry in the radiation belt as a function of Jovian longitude. Xiao and Thorne [23] studied the synchrotron radiation by using a bi-model loss cone distribution function and explained the radiation intensity, which peaks both at the equator and at high latitudes. Divine and Garret [24] estimated the charged particle flux from the observed radio emissions intensity spectrum which is sole estimation of radiation dose for Jupiter flyby and orbiter mission. It provides a quantitative model of charged particles between $1 \mathrm{eV}$ and several $\mathrm{MeV}$ based primarily on in-situ data returned from the pioneer and Voyager flybys of Jupiter. The in-situ data were supplemented by the Earth based radio telescope observations of synchrotron emission and theoretical conditions. Divine and Garret [24] model for energetic electrons in the inner Jovian magnetosphere has the ability to reproduce the observed pattern of synchrotron radiation. Levin et al. [25] compared the DG model with synchrotron emission observations and showed that DG model did not accurately describe the high-energy $(>1 \mathrm{MeV})$ electron population present in the Jupiter's inner radiation belt $(<4 \mathrm{RJ})$. Levin et al. [25] has constructed a computer model to simulate synchrotron emission from relativistic electrons trapped in the Jupiter's magnetic field. They suggested that higher order terms in the magnetic field coupled with relativistic beaming effects of synchrotron radiation, are primarily responsible for the observed rotational asymmetry.

In this paper, using recent data, we have computed synchrotronradiated power from electrons present in the Jovian magnetosphere. Earlier the studies had been done for the low frequency and the high frequency separately but we have taken the long-range trends in terms of energy emitted by the gyrating electrons interacting with the magnetic field. This energy range varies from a few $\mathrm{keV}$ to hundreds of $\mathrm{MeV}$. Section 2 discusses briefly theoretical formulation and properties of synchrotron radiation mechanism. Computational results are presented in section 3. Discussion of the results and conclusion of the present chapter is given in section 4 . The computational results of flux density have been plotted with frequency for various energies and compared with the recently observed radio spectrum. For the sake of comparative study we have also presented the earlier theoretical spectrum and observed spectrum along with our theoretical results and recent observed spectrum so as to have realistic interpretation of the measured decametric (DAM) power spectrum.

\section{Theoretical Formalism}

The gyrating charged particles radiate electromagnetic energy through the cyclotron process that, at high energy (relativistic) is known as synchrotron process. Schwinger [26] computed the radiated power via the rate at which the particle does work on the field of which it is the source. In this field components are not required and hence information about the polarization of the wave is not available. Westfold [20] derived electric field components from a relativistic gyrating charged particle and studied polarization and radiated power variation with frequency and other parameters of charged particles and medium.

The radiation from a single electron is totally polarized with the electric vector parallel to the radius of curvature of the orbit and in the case of an ultra- relativistic energy; the wave field is effectively restricted to a narrow cone of angular dimensions of the order of $[26,20,27]$

$$
\chi=\sqrt{1-\beta^{2}} \text { where } \vec{\beta}=\vec{v} / c, \vec{v} \text { is the velocity of charged particle. (1) }
$$

The electric field vector in this cone takes all directions transverse to the direction of emission. The method of Westfold [20] is pursued to evaluate the electric field by evaluating the trajectory of charged particle executing helical motion. Then Poynting vector is evaluated and averaged over gyration period to obtain average radiated power. The relativistic equation of motion of a charged particle having charge $e$ and mass $m$ in a uniform magnetic field $\mathrm{B}_{0}$ is written as

$$
\frac{d}{d t} \frac{m \vec{\beta}}{\sqrt{1-\beta}}=e \vec{\beta} \times \vec{B}_{0}
$$

This equation, using angular gyro frequency $\vec{\omega}_{H}=e / m \sqrt{1-\beta^{2}} \vec{B}_{0}$ can be separated in to parallel and perpendicular components as

$$
\begin{aligned}
& \dot{\beta}_{\prime}=0 \\
& \dot{\beta}_{\perp}=-\omega_{H} \times \vec{\beta}_{\perp}
\end{aligned}
$$

This shows that the motion of a uniform velocity along the magnetic field line and an uniform circular motion of radius $\beta_{\perp} c / \omega_{H}$ 
with the angular velocity $-\omega_{H}$ about the direction of $\vec{B}_{0}$. Taking the origin of the co-ordinates as the projection from the initial position of the electron on to the axis of the helix and $\alpha$ as the constant angle between $\vec{\beta}$ and $\vec{B}_{0}$, the equations of trajectory reduces to

$$
\frac{r}{c}=\frac{\beta}{\omega_{H}}\left[\left(\hat{i} \cos \omega_{H} t+\hat{j} \sin \omega_{H} t\right) \sin \alpha+\hat{k} \omega_{H} t \cos \alpha\right]
$$

Here, $\hat{i}, \hat{j}$, and $\hat{k}$ form a right handed orthogonal system of unit vectors such that $i$ is in the direction of the initial position and $k$ is in the direction of $\vec{B}_{0}$. Differentiating equation (3), the electron velocity is given by Westfold [20].

$$
\dot{\gamma}=\beta c \sin \alpha\left(-\vec{i} \sin \omega_{H} t+\vec{j} \cos \omega_{H} t\right)+\vec{k} \cos \alpha
$$

The acceleration is always along the principle normal to the trajectory. The radius of curvature has the constant value $\beta c /\left(\omega_{H} \sin \alpha\right)$ . The electric and magnetic vectors $\vec{E}$ and $\vec{B}$ at the point ' $s$ ' and instant ' $t$ ' are given in terms of position $r_{1}\left(t^{\prime}\right)$ of the retarded time $t$ ' by the relation Westfold [20]

$$
\vec{E}=\frac{\mu e c \vec{n} \times\left[\left(\vec{n}-\vec{\beta}^{\prime}\right) \times \dot{\vec{\beta}}^{\prime}\right]}{4 \pi R\left(1-\vec{\beta}^{\prime} \cdot n\right)^{3}} \text { and } \vec{B}=\frac{1}{c} \vec{n} \times \vec{E}
$$

where $\mu$ isthepermeabilityoffreespace, $\vec{n}=R\left(t^{\prime}\right) / R, \vec{\beta}^{\prime}=\dot{\gamma}_{1}\left(t^{\prime}\right) / c$ , $t^{\prime}=t-R\left(t^{\prime}\right) / c$ and $R\left(t^{\prime}\right)=r-r_{1}\left(t^{\prime}\right)$ The null directions of the fields are where

$$
\vec{n}=\vec{\beta}^{\prime} \pm \chi^{\prime} \frac{\dot{\vec{\beta}^{\prime}}}{\dot{\beta}^{\prime}}
$$

Equation (5) shows that the field vectors would be large when both $\chi^{\prime}$ is close to unity and $\theta$ the angle between $\beta^{\prime}$ and $\vec{n}$ is small. For a charged particle executing a periodic motion, the wave field spectrum would consist of a superposition of harmonics of the fundamental frequency $\omega_{H} / 2 \pi$.To calculate the average radiated power at any point, we consider $\vec{n}$ in the plane containing $B_{0}$ and initial velocity of the charge. Following Westfold [20] which uses modified Bessel functions and its recurrence relations, the $\mathrm{n}^{\text {th }}$ harmonic electric field $\mathrm{E}_{\mathrm{n}}$ and characteristics of polarization ellipse $\mathrm{L}_{\mathrm{n}}$ are written as

$$
\begin{aligned}
& E_{n}=\frac{\mu e c \omega_{H}}{4 \sqrt{3 \pi^{2} r}} \exp \left(i n \omega_{H} \frac{r}{c}\right) \frac{n}{\sin \alpha}\left(\chi^{2}+\Psi^{2}\right) K_{2 / 3}\left[\frac{n}{3 \sin \alpha}\left(\chi^{2}+\psi^{2}\right)^{3 / 2}\right] \vec{i} \\
& \left.+\vec{i} \psi\left(\chi^{2}+\psi^{2}\right)^{1 / 2} K_{1 / 3}(x) \vec{j} \cos \alpha-\vec{k} \sin \alpha\right) \text { where } x=\frac{n}{3 \sin \alpha}\left(\chi^{2}+\psi^{2}\right)^{3 / 2} \\
& \text { and }
\end{aligned}
$$

$$
L_{n}(\psi)=i \frac{\left(\chi^{2}+\psi^{2}\right)^{1 / 2}}{\psi} \cdot \frac{K_{2 / 3}(x)}{K_{1 / 3}(x)}
$$

where $K_{1 / 3}(x)$ and $K_{2 / 3}(x)$ are modified Bessel functions of argument $x$. The electric field and polarization are the function of the angle $\psi$ between the direction of observation $\vec{n}$ and the closest generator of the cone containing the directions of motion $\delta$. Bessel functions $K_{1 / 3}(x)$ and $K_{2 / 3}(x)$ are real and positive. Equation (7) shows that the polarization is elliptical with axes along parallel and perpendicular to the projection of $\vec{B}_{0}$ on the plane transverse to $\vec{n}$. The energetic charged particle moving along magnetic field executes periodic motion; hence the radiated power is distributed among the harmonics of the fundamental frequency. Applying Parseval's theorem to the formula for the Poynting vector, one obtains the average value of radiated flux density

$$
\begin{aligned}
& <S>=\frac{\omega_{H}}{2 \pi} \int_{0}^{2 \pi / \omega_{H}} S t \text { where } S=\vec{E} \times \vec{H} \\
& =\frac{\omega_{H} \vec{n}}{2 \pi \mu c} \int_{0}^{2 \pi / \omega_{H}} E^{2} d t \\
& =\frac{\vec{n}}{\mu c}\left(E_{0}^{2}+2 \sum_{1}^{\infty}\left|E_{n}\right|^{2}\right.
\end{aligned}
$$

The average flux density represents the average power flowing normally through the unit surface elements at the given distance from the origin. The average power radiated into the unit solid angle at distance ' $r$ ' is obtained as

$$
<P(\vec{n})>=\frac{1}{2} P_{0}(n)+\sum_{1}^{\infty}<P_{n}(n)>
$$

The contributions of $\mathrm{E}_{\mathrm{n}}$ can be separated into components parallel and perpendicular to the projection of $\vec{B}_{0}$ in the plane normal to $\vec{n}$ which can be designated by the subscript 1 and 2 . The mean power radiated in the frequency band between $f$ and $f+d f$ could be written as

$$
<\mathrm{P}_{\mathrm{f} 1}>=\frac{1}{2} \sqrt{3} \mu \mathrm{e}^{2} \mathrm{cf}_{\mathrm{HO}} \operatorname{Sin} \alpha \mathrm{F}^{(1)}\left(\frac{\mathrm{f}}{\mathrm{f}_{\mathrm{c}}}\right)
$$

and

$$
\begin{aligned}
& <\mathrm{P}_{\mathrm{f} 2}>=\frac{1}{2} \sqrt{3} \mu \mathrm{e}^{2} \mathrm{cf}_{\mathrm{H} 0} \operatorname{Sin} \alpha \mathrm{F}^{(2)}\left(\frac{\mathrm{f}}{\mathrm{f}_{\mathrm{c}}}\right) \\
& <\mathrm{P}_{\mathrm{f}}>\mathrm{df}=\frac{<\mathrm{P}_{\mathrm{n}}>\mathrm{df}}{\mathrm{f}_{\mathrm{H}}}
\end{aligned}
$$

where $\left\langle\mathrm{P}_{\mathrm{f}}\right\rangle=\left\langle\mathrm{P}_{\mathrm{f}}^{(1)}\right\rangle+\left\langle\mathrm{P}_{\mathrm{f}}^{(2)}\right\rangle$

$f_{C}=3 f_{H 0} \frac{\operatorname{Sin} \alpha}{2 \chi^{2}}, f_{H 0}=\frac{e B_{0}}{2 \pi m}$ gauss and radiating charged particle to be an electron, we obtain

$$
\begin{aligned}
& <\mathrm{P}_{\mathrm{f}}>=2.34 \times 10^{-25} \mathrm{~B}_{0} \operatorname{Sin} \alpha \mathrm{F}\left(\frac{\mathrm{f}}{\mathrm{f}_{\mathrm{c}}}\right) \mathrm{Wm}^{-2} \mathrm{~Hz}^{-1} . \\
& \mathrm{f}_{\mathrm{c}}=4.20 \mathrm{~B}_{0} \frac{\operatorname{Sin} \alpha}{\xi^{2}} \mathrm{MHz} \text { and } \mathrm{f}_{\mathrm{H} 0}=2.80 \mathrm{~B}_{0} \mathrm{MHz}
\end{aligned}
$$

Thus an electron of relativistic energy $E$, moving in magnetic field $B$ having pitch angle $\alpha$ radiates power in synchrotron mode which is given by

$$
\begin{aligned}
& P(f, E, B, \alpha)=2.34 \times 10^{-25} B \operatorname{Sin} \alpha\left(\frac{f}{f_{c}}\right) \int_{f / f_{c} E}^{\infty} K_{5 / 3}(x) d x \\
& \text { where } f_{c}=4.20 \gamma^{2} B \operatorname{Sin} \alpha \quad M H z, \gamma=1+\frac{1}{m c^{2}}
\end{aligned}
$$

$E$ is the kinetic energy of the electron, $m$ is the mass of electron and $c$ is the velocity of light. The above equation can be used to evaluate power radiated from an electron of given energy and moving in the presence of magnetic field. If radiating volume contains a large number of electrons having different energy, then the total radiated power will be the sum of radiation from all the electrons while summing the radiation it is assumed that the radiated power from electrons of different energy is in the same direction. Within these assumptions, the total power radiated from the radiating electron flux $N(E, \alpha) m^{-2} \mathrm{sec}^{-1}$ is written as in $\mathrm{Wm}^{-2} \mathrm{~Hz}^{-1} \mathrm{sec}^{-1}$

$$
P(f \varphi L)=\int_{E_{1}}^{E_{2}} d E \int_{\alpha_{c}}^{\pi / 2} P(E, f, B, \alpha) N(E, \alpha) d \alpha
$$

Where $\varphi$ is the magnetic latitude, $L_{J}$ is the McILwain parameter. Knowing the model of Jovian magnetosphere specifies the ambient magnetic field value.

The total radiated flux to be received on the ground neglecting the absorption loss during the propagation of wave from the Jupiter to the Earth is given by

$$
P(f)=\int \frac{P(f, \varphi, L)}{v R^{2}} d V W m^{-2} H z^{-I}
$$

where $R$ is the distance between radiating region and reception point, $R=4.0$ A.U. (Astronomical Unit) which is $6 \times 10^{11} \mathrm{~m}$ as taken in our theoretical computation while the experimental value of $\mathrm{R}$ is observed to be 4.04 A.U [9] $d V$ is the volume element and $v$ is the velocity of charged particle. 
Citation: Singh S, Singh AK, Singh RP (2015) Modeling Jupiter's Synchrotron Emission from Relativistic Electron Trapped in Jovian Magnetosphere. Hydrol Current Res 7: 218. doi:10.4172/2157-7587.1000218

Page 4 of 7

\section{Computational Results}

In order to explain the observed power received on the surface of the Earth and onboard spacecraft emitted from Jovian magnetosphere it is essential to know the density, energy and pitch angle distribution of radiating particles as well as magnetic field variation in the generation region. The magnetic field variation in the Jovian magnetosphere is assumed to be dipolar in nature [28]. The dipole magnetic field variation with latitude is Baumjohann and Treumann [29].

$$
B\left(\varphi, L_{J}\right)=B_{0}\left(1+3 \sin ^{2} \varphi\right)^{1 / 2} / \cos ^{6} \varphi \text { where } B_{0}=\frac{B_{E}}{L_{J}{ }^{3}}
$$

where $B_{0}$, is the equatorial magnetic field on the Jupiter's surface and $\mathrm{L}$ is the McIlwain shell parameter. For the computational work we have taken the variation of equatorial surface magnetic field as modeled to range from 2-14 gauss [30]. The $\mathrm{L}_{\mathrm{I}}$-value variation is taken from 1.35 to 3 . The detailed pitch angle distribution and its variation is not known properly, so we have assumed for simplicity that the pitch angle of the radiating electrons to be near to $90^{\circ}$. This approximation is taken in view of the numerical computations of Singh [27], where they have shown that the radiated power does not in general change appreciably for changes in pitch angle by $\pm 20^{\circ}$ in the vicinity of $90^{\circ}$. They have shown that an increase in pitch angle leads to an increase in radiated power, which becomes maximum for $\alpha=90^{\circ}$. This shows that an electron spiraling along magnetic field will have the maximum radiated power at the mirror point. Thus electrons with small pitch angle will have maximum radiated power at high latitudes where as electrons with pitch angle close to $90^{\circ}$ will radiate maximum power in the equatorial region. The radiation is confined equatorially in the region between 1.35 and $3.5 R_{p}$, with higher latitude lobes residing on field lines at $2.5 \leq$ $L_{I} \leq 3.5$. The earlier observational results show that the radiated power contains major portions coming from the equatorial region [8]. Using equation (11) the power radiated by a single electron as a function of electron energy and radiated frequency from a location (which specifies the magnetic field) is evaluated. The computed results for $L_{J}=1.5$ and $\phi=0$ (equator) is shown in Figure 2. They are shown for discrete frequencies between $30 \mathrm{MHz}$ and $100 \mathrm{MHz}$ and electron energy is varied from $0.2 \mathrm{MeV}$ to $100 \mathrm{MeV}$. It is clearly seen that the radiated power increases as the energy of the radiating electron increases showing a

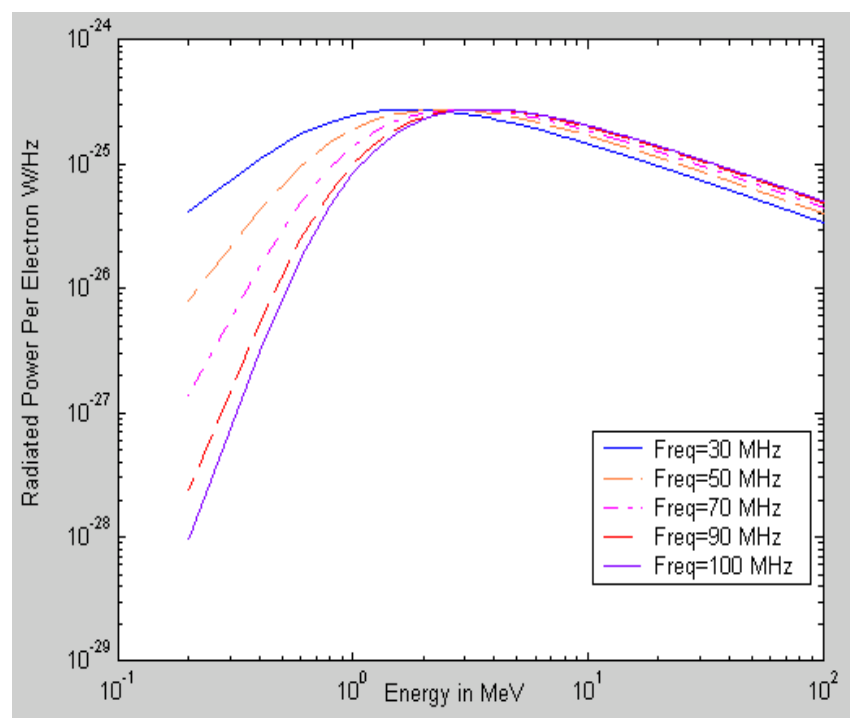

Figure 2: Theoretically computed radio spectrum between radiated power per electron with energy for different frequencies at equator and $L=1.5$. peak around 2-3 $\mathrm{MeV}$ and then decreases. The rate of increase is large as compared to the rate of decrease in radiated power .It is interesting to note also that below 2-3 $\mathrm{MeV}$ energy, the radiated power decreases with an increase in frequency whereas above this energy the same increases with frequency. However, the rate of decrease is so small that the spectrum almost appears more like a flat spectrum.

As the electron moves along dipolar magnetic field lines away from the equatorial zone towards the surface of Jupiter, the magnetic field increases. Figures $3 \mathrm{a}$ and $3 \mathrm{~b}$ consecutively shows the electrons from the place defined by $L_{J}=1.5$ and $\varphi=0^{\circ}, 15^{\circ}$. It is seen that the radiated power from electrons having energy in the range $50 \mathrm{MeV}-100 \mathrm{MeV}$ is approximately same at $300 \mathrm{GHz}$. The pattern of variation remains the same as depicted in Figures $4 \mathrm{a}$ and $4 \mathrm{~b}$ also, when $\mathrm{L}_{\mathrm{J}}$-value increases the

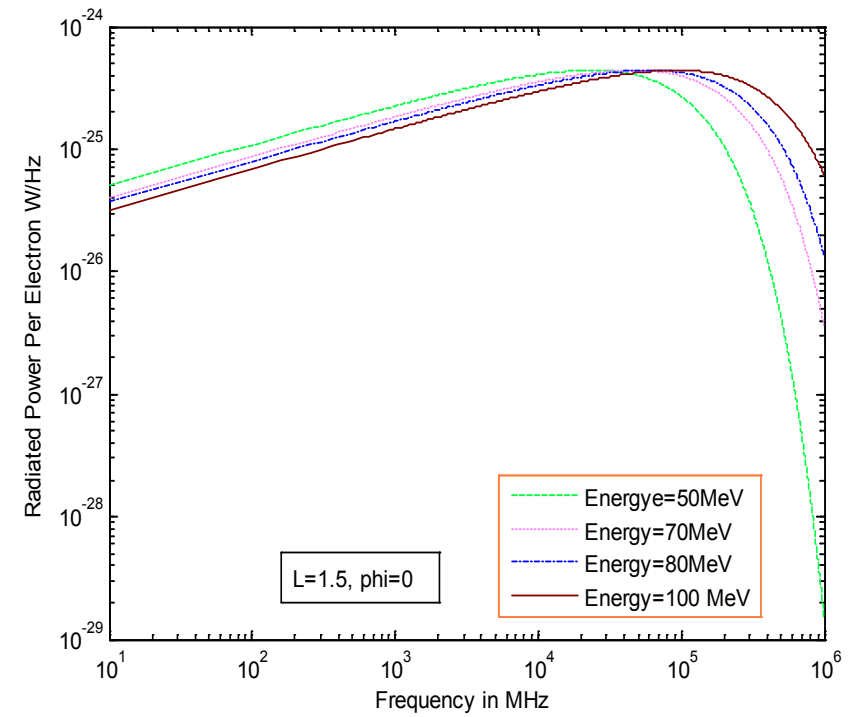

Figure 3 (a): Theoretically computed radio spectrum between radiated powe per electron with frequency for different energy at different parameters.

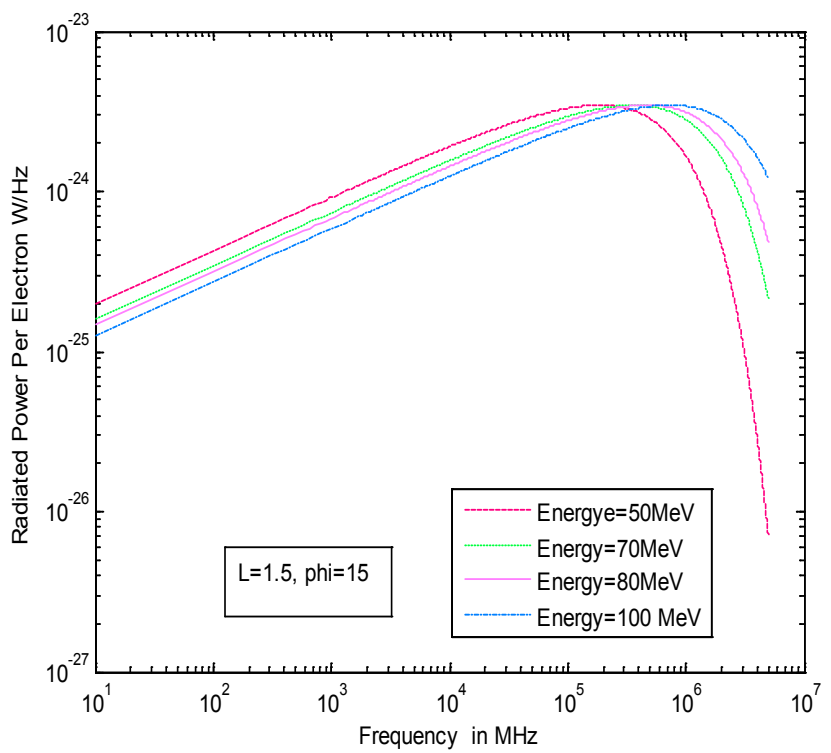

Figure 3 (b): Theoretically computed radio spectrum between radiated powe per electron with frequency for different energy at different latitude. 
equatorial magnetic field decreases. To understand the role of magnetic field on the radiated spectrum (both power radiated and frequency range) we have also computed power radiated from single electron located at $L_{J}=3.5$ and $\varphi=0^{\circ}, 15^{\circ}$. The results are shown in Figures $4 \mathrm{a}$ and $4 \mathrm{~b}$.

The power radiated from energetic electrons as it moves along the field line is computed for different $\mathrm{L}$-values and $\phi$. The results are not shown because nature of variation remains almost the same. The radiated power maximizes from the higher latitudes as compared to the lower latitudes. However, the rate of increase varies with frequency

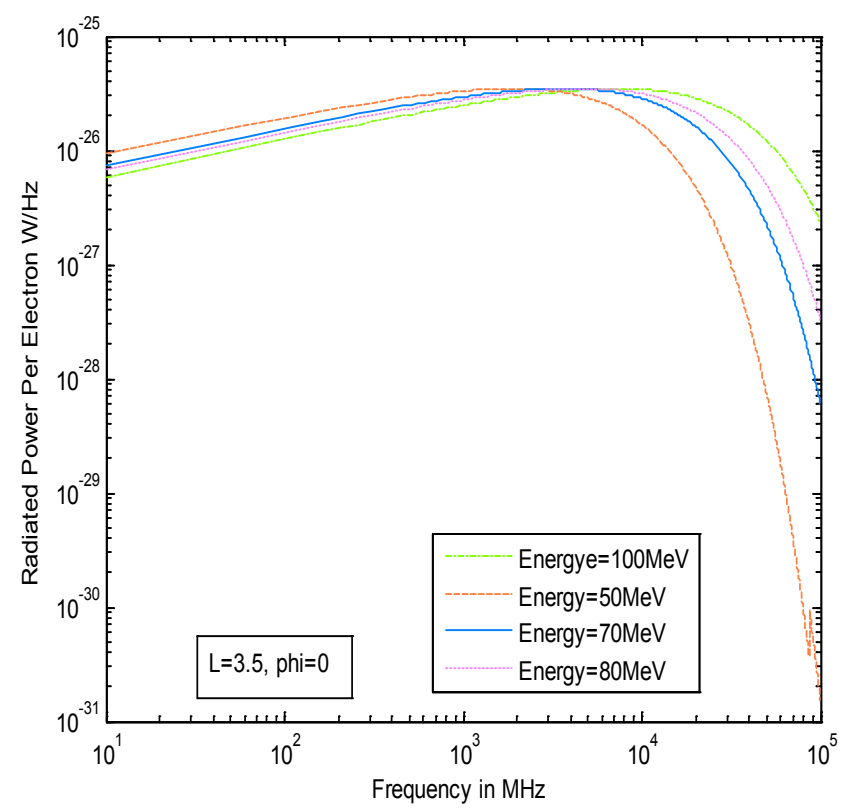

Figure 4 (a): Theoretically computed radio spectrum between radiated power per electron with frequency for different energy at equator and $L=3.5$.

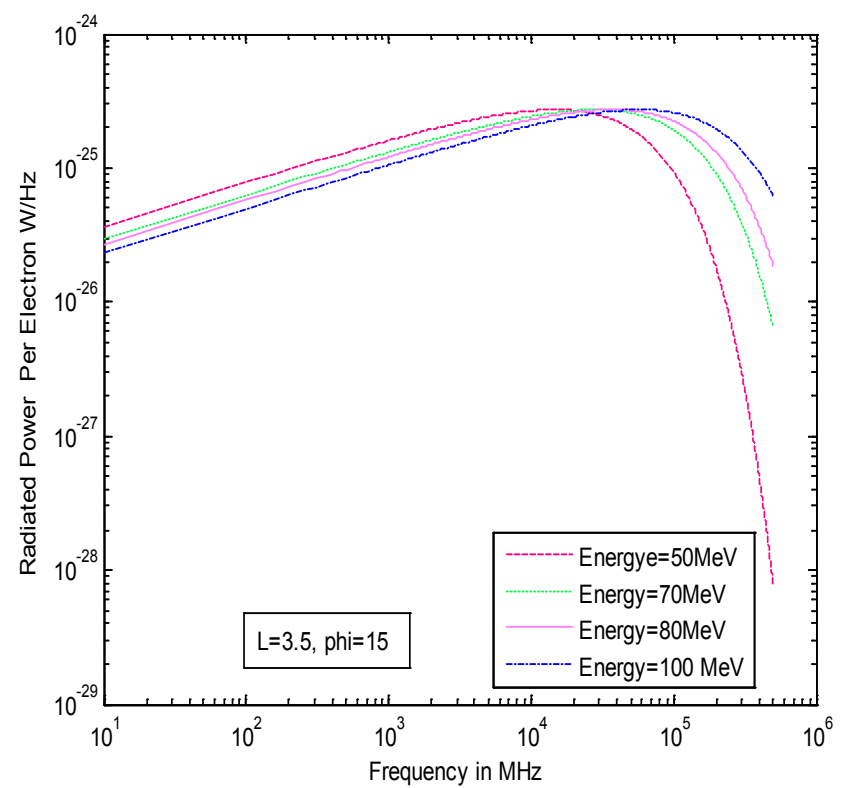

Figure 4 (b): Theoretically computed radio spectrum between radiated power per electron with frequency for different energy at some magnetic latitude and $L=3.5$. as well as energy of the electron. The power radiated from energetic electrons as it moves along the field line is computed for different $\mathrm{L}$-values and $\phi$. The results are not shown because nature of variation remains almost the same. The radiated power maximizes from the higher latitudes as compared to the lower latitudes. However, the rate of increase varies with frequency as well as energy of the electron.

The synchrotron power radiated by an electron in the entire energy range is minimum at the geomagnetic equator and increases rapidly as electron moves away from the equatorial region. For constant magnetic field, the radiated synchrotron power varies with electron energy and becomes maximum for certain electron energy. The synchrotron radiation from an electron is in the form of electromagnetic pulses radiated along the instantaneous velocity vector and lasts for very short duration. The radiated pulse along certain direction are separated by $\Delta T=2 \pi / \omega_{H}, \omega_{H}$ is the gyro frequency [27]. If the gyrofrequency of the medium is high, the time interval is small and more electromagnetic pulses are radiated per second. For example at $\mathrm{L}=3$ and $\phi=0^{\circ}, \omega_{H}=20450 \times 2 \pi, \Delta T=4.88 \times 10^{-5} \mathrm{Sec}$ and $\varphi=30^{\circ}$, $\omega_{H}=4.5 \times 10^{9} \times 2 \pi$, and $\Delta T=2.22 \times 10^{-10} \mathrm{sec}$. Thus the number of pulses at some latitude is quite large as compared to the equatorial value. This leads to the conclusion that the cyclotron radiated power increases with increasing magnetic field. This supports present computational results. In evaluating the equation, one requires either the measured electron energy spectrum or a model for the same. Singh et al. [7] prepared the flux variation of mildly relativistic electrons with $\mathrm{L}_{\mathrm{J}}$-value on the basis of available measurements. They have considered the energy range $40 \mathrm{keV}<E<5 \mathrm{MeV}$. de Pater (2004) has modeled the latitude and energy distribution of Jupiter's electron flux, where the constants have been chosen to fit the observed synchrotron radiation spectrum. Xiao and Thorne [23] considered bi-model electron distribution to explain observed peaks in the radiation intensity both at the equator and at high latitude. In the present computation, we have considered the synchrotron radiation by an electron is many times more effective in the high latitude as compared to the synchrotron radiation by the electron in the equatorial region.

Some more computational plots taking the variation of radiated power per electron with latitude for different frequencies has been done to understand the dynamics of the gyrating electrons. To see the effect of emitted frequency we have computed synchrotron radiated power per electron from Jupiter at a different frequency and varying other parameters which is shown in Figures $5 \mathrm{a}$ and $5 \mathrm{~b}$. From this Figure we observed that the synchrotron-radiated power per electron is minimum for equator then increases with latitude and shows a maximum value at $30^{\circ}$ and then decreases gradually to minimum at $60^{\circ}$.From both the From the Figure 5 we observed that the radiated power per electron decreases gradually with increasing the emitted frequency for fixed energy and $\mathrm{L}_{\mathrm{I}}$-value. The plots for different frequency are evenly spaced and show maxima at $30^{\circ}$ geomagnetic latitude. The total radiated flux to be received on the ground from the Jupiter to the Earth have been computed for different frequencies and shown in Figure 6 for energies $100 \mathrm{MeV}$ and $200 \mathrm{MeV}$. To compare our theoretically computed results with the observed radio spectrum we have included some old and recent experimental power frequency spectra for observed deccametric emission which is shown in Figure 6. The Figure shows that the nature of experimental observation of Smith and Desch [31,32] as well as recent experimental observations of Imke De Pater and Bolton et al. $[8,9]$ are consistent with our computed synchrotron flux. In the Figure 6 we have also included the theoretically computed synchrotron flux by Singh et al. [7]. After comparing our results we found that our 


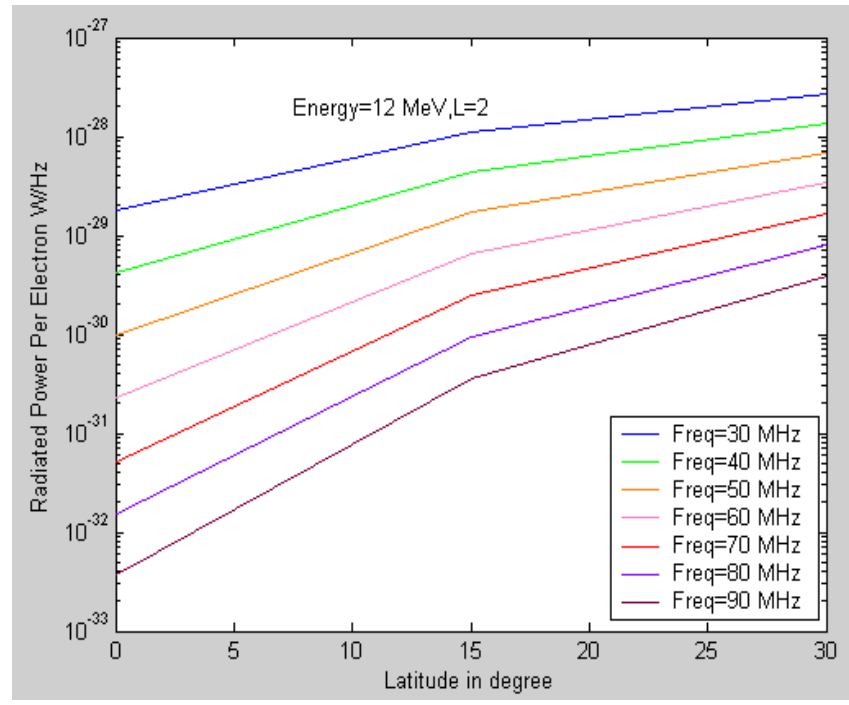

Figure 5 (a): Computed radio spectrum between radiated power per electron with Latitude for different frequencies and at a fixed energy at equator and $L=2$.

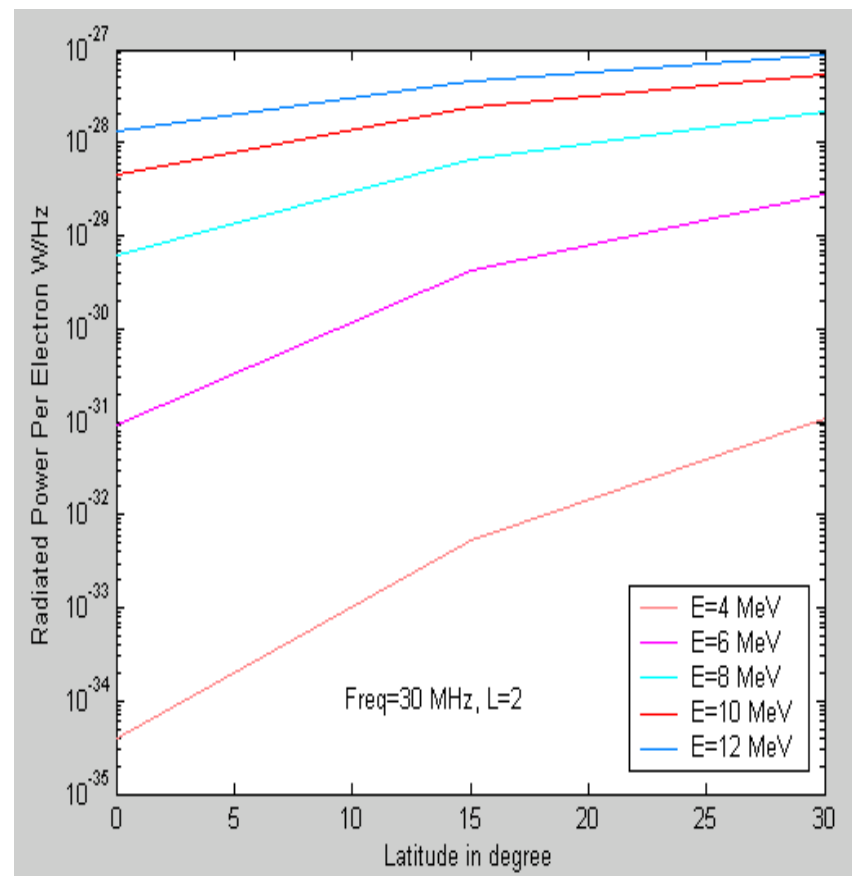

Figure 5 (b): Computed radio spectrum between radiated power per electron with Latitude for different energies and at a fixed frequency $L=2$.

computed flux density are much more comparable with the recent observed spectrum, of Imke de Pater and Bolton et al.[12,9] whereas the computation results of Singh et al.[7] compares well with the old observations of Desch and Carr [32] and Smith et al. [31]. Only there is less than one order of discrepancy in recently observed flux density and our computed flux density, otherwise both nature and range of computed flux density compares well with the observed spectrum.

\section{Discussions and Conclusion}

In this study, we have computed several parameters for synchrotron radiation emitted by the gyration of relativistic electrons trapped in

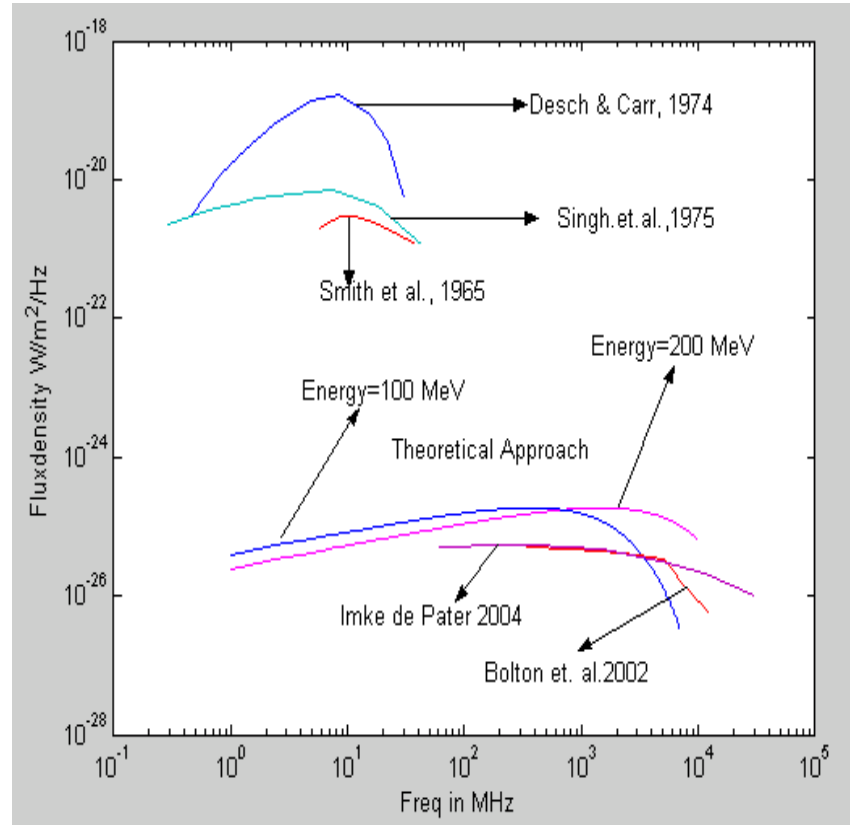

Figure 6: The figure inculcates the various experimental and theoretically computed flux density spectrums with frequency and could be seen with a good correlation.

the inner Jovian magnetosphere. The most widely accepted theory postulates that the relativistic electron originate in the solar wind or in the outer Jovian magnetosphere and the diffuse inward by gaining energy through the conservation of first and second adiabatic invariants $[33,34]$. This accounts for $10 \mathrm{MeV}$ electrons near the peak of synchrotron zone. Our comparison of flux density is quite consistent with the recent observed radio spectrum observed by Cassini fly-by over Jupiter [9]. We see that the general nature of curve remains unchanged by changing the flux of energetic electrons. The synthesis of these computations leads us to conclude that the general shape of power spectra recorded by Cassini fly-by is governed by the detailed nature of the energetic electron spectrum. The maximum flux density of $10^{-26} \mathrm{~W} \mathrm{~m}^{-2} \mathrm{~Hz}^{-1}$ lies around frequency $1 \mathrm{GHz}$ for energy $100 \mathrm{MeV}$ and $2 \mathrm{GHz}$ for $200 \mathrm{MeV}$, which is in close consistency with observed radio spectrum. In fact one-to-one correspondence of the measured power spectrum cannot be brought about because of the lack of detailed energy spectrum and spatial distribution of the energetic electrons in the Jovian ionosphere and magnetosphere. The present theoretical work supports the observation of Bolton and Imke de Pater [9, 12] Xiao and Thorne [23] by using a bimodal loss cone distribution function has shown that the variance of synchrotron radiation with latitude depends on the electron anisotropy, for low anisotropy, synchrotron radiation increases with latitude and reaches a maximum at the particle mirror points: for high anisotropy, it decreases with latitude and maximizes at the equator. Thus, his model suggested explaining the radiation intensity, which peaks both at the equator and at high latitudes. Warwick [35] has shown that the flux density in the dynamic spectra of Jupiter exceeds $10^{-21}$ or even $10^{-20}$ $\mathrm{Wm}^{-2} \mathrm{~Hz}^{-1}$ at the frequency of $39 \mathrm{MHz}$ is also not consistent with Desch and Carr's image of power spectrum. The general feature of computed power spectrum is found to depend on the flux of relativistic electrons, which are believed to be produced by the acceleration of electrons by ' $I O$ ' sheath. The orbital position of ' $I o$ ' influences the radiating electron flux and thereby affects the radiated power flux [7]. Some more study related to flux density could be done for higher energy $\leq 20 \mathrm{MeV}$ to see if the 
Citation: Singh S, Singh AK, Singh RP (2015) Modeling Jupiter's Synchrotron Emission from Relativistic Electron Trapped in Jovian Magnetosphere. Hydrol Current Res 7: 218. doi:10.4172/2157-7587.1000218

range of flux density changes to what extent to explain more clearly the DAM power spectrum.

Efforts should be made to model radial diffusion at Jupiter's which produces accurately the synchrotron emission radio spectrum and also the study of electron energization that are known to occur in Earth's magnetosphere accelerating the electron by wave particle interactions and field aligned potential in auroral zone. The mechanisms, which combine radial diffusive transport with wave particle interactions such as recycling and magnetic pumping, could be proposed. The fast interplanetary shock and Earth's magnetosphere has been proposed as a source of drift resonance acceleration to explain the sudden appearance of relativistic electrons in terrestrial Van Allen radiation belts. A similar process could exist at Jupiter although analogies between both are not always appropriate. Thus, the inner radiation belts of Jupiter and Earth both contain extremely high-energy electrons that require substantial acceleration by the process other than adiabatic radial diffusion.

\section{Acknowledgement}

Shubha Singh is thankful to Council of Scientific and Industrial Research (CSIR) for providing financial support to carry on the research work smoothly. My special thanks go to Mr. Surendra Prasad for his help in computation.

\section{References}

1. Burke BF, Franklin KL (1995) Observation of a variable radio source associated with the planet Jupiter. J. Geophys Res 60: 213-217.

2. Sloanaker RM, Boland JW (1961) Observations of Jupiter at a wavelength of 10 cm. Astrophys. J 133:649-656.

3. Field GB (1961) Can the Jupiter emission be cyclotron radiation? Astron J 66 283

4. Chang DB, Davis L Jr (1962) Synchrotron radiation as the source of Jupiter's polarized decimeter radiation. Astrophys. J.136: 567-581.

5. Berge GL, Gulkis S (1976) Earth based radio observations of Jupiter: millimete to meter wavelengths. In. Gehrels, T. (Ed.,) Jupiter. University of Arizona Press, Tucson : 621-622.

6. Warwick JW (1960) Relation of Jupiter's Radio Emission at long wavelengths to solar activity. Science 132: 250-1252.

7. Singh RN, Singh RP, Rai RS (1976) Synchrotron Process as the source of deccametric radiation from Jupiter. Ind. J. Rad. Spa. Phys., 5: 285-288.

8. de Pater I (2004) Lofar and Jupiter's radio (Synchrotron) emissions. Planet. Space Sci 52: 1449-1454.

9. Bolton SJ, Janssen M, Thorne R, Levin S, Klien M, et al. (2002) Ultra-relativistic electrons in Jupiter's radiation belts. Nature. 415: 987-991.

10. de Pater I, Dunn DE (2003) VLA observations of Jupiter's synchrotron radiation at 15 and $22 \mathrm{GHz}$, ICARUS 163: 449-445.

11. Sault RJ, Oosterloo T, Dulk GA, Leblanc $Y$ (1997) The first three-dimensional reconstruction of a celestial object at radio wavelengths: Jupiter's radiation belts, Astron. Astrophys 324: 1190-1196

12. Kloosterman LA, Bryan Butler B, Imke de Pater I (2008) VLA observations of synchrotron radiation at $15 \mathrm{GHz}$, Icarus 193: 644-648.

13. Santos-Costa D and Bolton SJ (2008) Discussing the processes constraining the Jovian synchrotron radio emission's features, Planet. Space Sci 56: 326-345.

14. Ezoe Y, Ishikawa K, Ohashi T, Miyoshi Y, Terada N, et al. (2010) Discovery Of Diffuse hard X-ray emission around Jupiter with Suzaku, The Astrophysical Journal Letters 709: L178-L182.

15. Horne RB, Thorne RM, Glauert SA, Menietti DG, Shprits YY (2008) Gyroresonant electron acceleration at Jupiter, Nature Physics 4: 301-304.

16. Tsuchiya F, Misawa H, Imai K, Morioka A (2011) Short-term changes in Jupiter's synchrotron radiation at $325 \mathrm{MHz}$ : Enhanced radial diffusion in Jupiter's radiation belt driven by solar UV/EUV heating, J. Geophys. Res, 116.

17. Thorne KS (1965) Dependence of Jupiter's decimeter radiation on the electron distribution in its Van Allen Belts. Radio Sci. 69: 1557-1560.
18. Roberts JA (1976) The pitch angles of electrons in Jupiter's radation. Astronomical Society of Australia, Proceedings 3:53-55.

19. Thorne KS (1963) The theory of synchrotron radiation from stars with dipole magnetic fields. Ap J Supp 81: 1-51.

20. Westfold KC (1959) The polarization of synchrotron radiation. Astrophys. J 130 : 241-258.

21. De Pater I (1981) A comparison of the radio data and model calculations of Jupiter synchrotron radiation. The high-energy electron distribution in Jupiter's inner magnetosphere. J. Geophys. Res. 86: 3397-3422.

22. De Pater I (1981) A comparison of the radio data and model calculations of Jupiter's synchrotron radiation. East- West asymmetry in the radiation belts as a function of Jovian longitude. J. Geophys. Res, 86: 3423-3429.

23. Xia F, Thorne MR (2006) Assesment of Jovian synchrotron radiation with a bimodal electron population. Planet Space Sci 54: 405-409.

24. Divine N, Garret HB (1983) Charged particle distribution in Jupiter's magnetosphere. J. Geophys. Res 88: 6889-6903.

25. Levin MS, Bolton JS, Gulkis SL, Klien JM, Bhattacharya B, et al. (2001) Modeling Jupiter's synchrotron radiation. Geophy. Res. Lett., 28: 903-906.

26. Schwinger J (1949) On the classical Radiation of accelerated electrons, Phys. Rev 75:1912-1925.

27. Singh RN, Singh RP (1968) Study of synchrotron radiation from energetic electrons in the magnetosphere. Ann. Geophys 4: 991-998.

28. Carr TD, Desch MD, Alexender JK (1983) Phenomenology of magnetospheric radio emissions, In:Physics of Jovian Magnetosphere. Ed A.J. Dessler Cambridge University Press. New York. 226-284.

29. Baumjohann W, Treumann RA (1999) Basic Space Plasma Physics, Imperia College Press.

30. Connerney JEP (1993) Magnetic fields of the outer Plantes, J. Geophys. Res 98: $18659-18679$

31. Smith AG, Lebo GR, Six NF, Carr TD, Bollhagen H, et al (1965) Decameterwavelength observations of Jupiter-the apparitions of 1961 and 1962 Astrophys. J 141: 457-477.

32. Desch MD, Carr TD (1974) Dekametric and hectometric observations of Jupite from the RAE-1 satellite. Astrophys. J.Let 194: 57-59.

33. Coroniti FV (1975) The magnetosphere of Earth and Jupiter (Ed.,) Formisano V, Reidel Dordrecht: 391-410.

34. de Pater I, Goertz CK (1990) Radial diffusion models of energetic electrons and Jupiter's synchrotron radiation 1 . Steady state solution. J. Geophys. Res 95: 39-50.

35. Warwick JW (1967) Radiophysics of Jupiter. Space Science Reviews 6: 841 891. 